\title{
Radio Resource Management for Green Wireless Networks
}

\author{
Cristina Comaniciu \\ ECE Department \\ Stevens Institute of Technology \\ Hoboken, NJ \\ e-mail: ccomanic@stevens.edu
}

\author{
Narayan B. Mandayam \\ WINLAB, Department of ECE \\ Rutgers University \\ New Brunswick, NJ \\ e-mail:narayan@winlab.rutgers.edu
}

\author{
H. Vincent Poor \\ EE Department \\ Princeton University \\ Princeton, NJ \\ e-mail: poor@princeton.edu
}

\begin{abstract}
In this paper, an auctioning strategy is proposed for cellular networks that ensures net energy savings. The pricing scheme, in conjunction with the two dimensional bid structure, incentivizes cooperation at the terminal nodes for better interference management at receivers and for cooperative relaying. It is shown that, for the proposed auctioning strategy, network operators are guaranteed revenue gains, mobile nodes' dominant strategy is to bid their true valuation of their energy resources, and overall effective energy gains occur under the assumption of a reserve price for bidding.
\end{abstract}

Keywords-component; pricing, Vickrey auction; cooperative relaying; interference management, cellular, green radio.

\section{INTRODUCTION}

Climatologists have determined that the accumulation of greenhouse gases (primarily carbon dioxide $-\mathrm{CO}_{2}$ ) in the upper levels of the earth's atmosphere is forming a shield that prevents heat rays escaping from the earth, thereby warming the planet to unnatural levels $[1,2]$. Scientists have warned that such global warming may have significant negative impact on global health, social and economic wellbeing. One measure that objectively quantifies the impact of modern civilization on global warming is the carbon footprint, which measures the quantity (in pounds or kilograms) of $\mathrm{CO}_{2}$ released into the atmosphere by a certain human activity. Greenhouse gas (GHG) emissions have risen sharply with the industrial revolution and they are projected to further increase dramatically if significant measures are not taken to prevent this. Currently, the telecommunication industry sector accounts for about $2 \%$ of the GHG footprint (about the same percentage as air traffic) [4]. For the wireless communications, most of the significant environmental impact is due to high energy consumption in cellular base stations (BSs) [3].

There is considerable current interest in reducing the energy consumption in the wireless sector, particularly in Europe, with companies such as Orange (France), Erricson (Sweden) and Vodaphone (UK) vouching to dramatically reduce their $\mathrm{CO}_{2}$ emissions in the range of $50-80 \%$ by 2020 [3]. Further, significant effort has been directed toward the design of base stations powered by alternate energy sources (e.g. solar cells and wind turbines).

Although research on the design of green radio solutions is still in its infancy, significant results already exist on energy efficient designs to conserve battery power for mobile terminals and wireless sensors. Because of the focus on mobile terminals, this prior work has primarily addressed uplink communications. However, from an overall perspective, it is necessary to consider the downlink, as base stations are the primary energy consumers of cellular networks. Consequently, in this paper, we approach energy efficiency from the perspective of optimized downlink communications, with a direct objective of reducing energy consumption at the base station. We conjecture that cooperation between end users and network operators may result in significant overall energy savings. We propose an auction based pricing system that incentivizes both parties to cooperate for energy savings, and we prove that a game theoretic model for this auction admits a Nash equilibrium at which the network operator rewards cooperative behavior of mobile terminals, while the terminals have a dominant strategy of bidding their true valuation of energy resource.

In this formalism, the nodes' cooperative options include employing better signal processing/interference cancellation techniques, flexible QoS requirements, and relaying packets for remote nodes.

\section{ENERGY MODELS FOR CELLULAR}

\section{A. Energy based Utility Models for Base Station}

According to references [3,5], a significant part of the energy consumption for network operators is the operating energy for the base stations (as opposed to the embodied energy for manufacturing, as it is in the case for mobile terminals), with about $57 \%$ of the energy consumption being used for radio access. It is thus apparent that efficient resource management algorithms are key components for more efficient communications.

By analyzing the base station power model consumption in [5], we can see that significant energy is consumed in transmit power, which results in even higher energy consumption due to the inefficiency of the linear power amplifiers (LPAs). Consequently, according to [5], for each watt of output power, approximately $3.5 \mathrm{~W}$ are required at the LPA input. Consequently, the effective gains in reducing output power at the base station need to be multiplied by the inverse of the LPA efficiency at the base station, $\eta_{\mathrm{B}}$.

As $3 \mathrm{G}$ services mature, the revenue of mobile network operators is expected to grow at a much slower rate than the corresponding increase in the amount of data traffic [3], with the starkest difference seen so far with the companies that 
offer more aggressive pricing for data services [3]. One way to improve revenues for the operators is to reduce their energy costs, so that becoming "greener" for environmental reasons is also financially beneficial for network operators.

Assuming an effective cost per unit energy to be $c$, the cost of transmitting 1 bit for a connection can be expressed as ( similarly to the energy per bit utility function for mobile terminals proposed in [8]):

$$
C_{b}=\frac{\eta_{B} P_{t} c E[K]}{r}=\frac{\eta_{B} P_{t} c}{r P_{c}(\gamma)},
$$

where $r$ is the transmission rate, $E[K]=$ average number of required retransmissions per packet, and $P_{c}(\gamma)$ is the probability of correct reception of a packet, with $\gamma$ being the signal-to-interference-plus-noise ratio (SINR) at the receiving node. $P_{c}(\gamma)$ is determined by the receiving node characteristics (e.g., current interference and noise levels, interference cancellation capabilities, etc.) and on the node's QoS specifications (e.g. transmission rate, modulation level, etc.).

The cost in (1) can be reduced by:

(i) better interference management at receiving nodes,

(ii) reduced QoS requirements in terms of transmission rates, or

(iii) support of external terminals for relaying, with an effect of reducing $P_{t}$, by using a smaller effective transmission range for the base station.

From the above discussion, it is apparent that cooperation from mobile terminals may potentially result in gains for the network operators. One way to incentivize such cooperation is to introduce reward pricing for cooperating nodes (see for example [12]). These rewards will encourage the nodes to trade off their QoS requirements or energy (for the case of relaying nodes) for monetary rewards from the system operator. We note that in designing such a pricing scheme, the green energy savings goal is to reduce the overall energy consumption per transmitted bit, rather than simply move the energy burden from the base station (BS) to the mobile terminals.

The network operator's utility per transmitted bit is proportional to the revenue obtained from the transmission. A higher transmission power will result in higher energy costs, while a higher price per unit energy $\lambda$ for the end user, will result in more revenue. A reimbursement price per bit $R$ can be used to reward intermediate nodes that facilitate transmission with reduced energy costs. Thus the utility function perceived by the base station operators is a measure of revenue per bit and can be expressed as (see also [12] for a similar definition)

$$
U_{B S}=E_{b}(\lambda-c)-R,
$$

where $E_{b}$ is the energy per transmitted bit consumed by the BS: $E_{b}=\eta_{B}\left(P_{t}\right) P_{t} / r P_{c}(\gamma)$, and $R$ is the monetary reward for relaying which could be expressed as $R=E_{b}{ }_{b} \mu$, with $E^{\prime}{ }_{b}=$ energy per bit consumed by a relaying node, and $\mu=$ unit price per energy expenditure.
We can see that $E_{b}$ can be reduced by either reducing the required throughput, improving the packet success rate (better interference management), or reducing the transmitted power (e.g., using relays).

A profit margin $\varepsilon$ per unit energy expenditure for the BS can be defined, which will guarantee the network operator's revenue in the absence of helper terminals. Consequently, the price charged by the operator for the unit energy expenditure per bit is given by $\lambda=c+\varepsilon$. Hence, the BS utility function can be written as

$$
U_{B S}=E_{b} \varepsilon-E_{b}^{\prime} \mu .
$$

\section{B. Utility Models for Mobile Terminals}

Two different terminal types should be considered for modeling: (i) the intended receiver (destination node); and (ii) a relaying terminal.

\section{(i) Destination node}

At the receiving node, the utility of the terminal measures the level of satisfaction a customer gets from the service it receives. A general valuation function $\Gamma\left(T_{D}, \lambda E_{b}, q\right)$ is proposed to capture the following performance metrics that are relevant to the destination terminal: throughput $\left(T_{D}\right)$, price charged by the BS per correctly received bit $\left(\lambda E_{b}\right)$, and energy expenditure for advanced signal processing $(q)$. The function $\Gamma$ should be: (i) monotonically increasing in throughput $T_{D}$; (ii) monotonically decreasing in $\lambda E_{b}$ and $q$.

Consequently, the destination node's utility can be determined to be equal to its valuation function:

$$
U_{D}=\Gamma\left(T_{D}, \lambda E_{b}, q\right) \text {. }
$$

The selection of function $\Gamma$ will determine the relative preferences given by a user to these three metrics. Given the price $\lambda$ announced by the BS, and the user's profile parameters, the destination terminal optimizes its utility function, for a set of choices in the following areas: signal processing algorithms for better interference management at the receiver, and QoS specifications in terms of throughput requirements for the connection.

The destination node can trade off required throughout for lower payment, or can choose to implement sophisticated signal processing at the receiver (e.g. multiuser detection, diversity, beamforming, etc.) to reduce the interference level with a direct impact in reducing the required transmission power for the base station (for a given SINR requirement). The latter choice comes with a price of increased computational energy expenditure, which also affects the terminal's utility.

The choice of the function $\Gamma$ is not unique. Future work will consider the impact of this function definition on capturing the real preferences of the destination user, and on impacting the achievable performance metrics at user and network level. 


\section{(ii) Relaying node}

Using relaying nodes can be beneficial for reducing the overall energy consumption of the transmission. The operator can reward cooperative behavior of the nodes, by paying a reimbursement price for the energy expended by relaying terminals.

The utility of a relaying terminal will depend on the reward gained $R$, and on the energy expenditure of the terminal $E^{\prime}{ }_{b}$, through the valuation function $\theta\left(E^{\prime}{ }_{b}, c\right)$ :

Definition: The valuation function $\theta\left(E^{\prime}{ }_{b}, c\right)$ is the minimal reward price the potential relaying terminal would accept to be paid for its energy expenditure $E^{\prime}{ }_{b}$.

An example of a choice for the valuation function for a user could be $\theta\left(E_{b}, c\right)=E^{\prime}{ }_{b} \mu$. Users may also be able to set preferences to enable or disable relaying based on their current battery levels. As such we define a utility function for the relaying terminal to be

$$
U_{R}=\left\{\begin{array}{l}
-\infty, \quad \text { if } E_{r}<E_{t h}, \\
R-\theta\left(E_{b}^{\prime}, c\right), \quad \text { ow }
\end{array}\right.
$$

where $E_{r}$ is the current battery level for the potential relaying node, and $E_{t h}$ is the threshold set by the user's preferences: $E_{t h} \in(0,1]$, with a value of 1 being assigned by a user who is not interested in monetary rewards and will not cooperate in relaying.

\section{AN AUCTION BASED GAME FOR ENERGY SAVINGS}

The network operator can potentially gain additional revenue and save energy by enlisting the support of helper nodes for relaying. We propose an auction based mechanism for selecting a relaying node for a given connection.

\section{A. Auction Mechanism}

An auction is a negotiation mechanism or a set of trading rules for exchange of goods between sellers and buyers. Many auctioning mechanisms have been studied by the economics community under various conditions and mechanism rules. These various auctioning mechanisms lead to a variety of properties and outcomes of the bidding process. Some important properties to be specified in an auction are: (i) open versus sealed-bid (bids are known or not); (ii) number of bids allowed (one time bidding versus successive bidding), (iii) winning rules, and (iv) payment (e.g. highest price, second highest price, etc.).

There is a rich literature of applying auctioning strategies for resource allocation problems in ad hoc networks. Some recent results address auction design for cooperative relaying with a solution based on best response bid updates for an SNR and a power auction solution, respectively [6].

For the case of dynamic resource allocation problems, one time bidding with sealed-bids reduces drastically the amount of information needed for bidding, and hence reduces overhead and resource allocation delays.

For these kind of applications, the Vickrey auction [10] is an appealing choice, as it has been shown to have desirable properties such as a dominant equilibrium strategy for the bidders to bid their true valuation of the goods. In a Vickrey auction, the highest bid wins, but pays the second-highest price.

For our relaying allocation problem, we consider a more complex two-dimensional bidding, in which bidders specify the estimated energy gains they provide for the base station, and their monetary reward requests. As such, a bidder $i$ will specify its bid as $\mathbf{s}_{i}=\left(\Delta \hat{E}_{b}(i), R(i)\right)$.

The base station receives all the bids from all users and computes its utility gain from selecting node $j$ as

$$
\Delta U_{B S}(j)=\Delta \hat{E}_{b}(j) \mathcal{E}-R(j)
$$

The base station considers for final selection only those bids for which its utility gain (6) is positive. The base station's utility gain is maximized by selecting an auction winner node $j^{*}$, such that

$$
\begin{aligned}
j^{*} & =\left.\underset{j}{\arg \max } \Delta U_{B S}(j)\right|_{\Delta U_{B S}(j)>0} \\
& =\left.\underset{j}{\arg \max } \Delta U_{B S}(j)\left\{\Delta \hat{E}_{b}(j) \varepsilon-R(j)\right\}\right|_{\Delta U_{B S}(j)>0}
\end{aligned}
$$

As we have mentioned previously, a Vickrey auction implementation is preferable, due of its desirable equilibrium property: all users have a weakly dominant strategy of bidding their own valuation of the resources.

For the Vickrey auction implementation, the equilibrium strategy for an arbitrary bidder $j$ is to select its reward $R(j)$ to be $R(j)=\theta(j)$.

Theorem 1: For the proposed second price Vickrey auction with two-dimensional bids, paying the second highest price for the winner may lead to gains less than the second highest utility gain. Furthermore, the auction pricing strategy does not guarantee positive utility gains for the base station.

Proof: Consider user $j$ to be the winner of the auction, user $i$ to be a user that has requested the next highest price (but not necessarily yielding the next highest gains) and user $k$ that yields the second highest utility gain for the base station.

Since node $k$ yields the second highest utility gain, we have

$$
\begin{aligned}
& \Delta E_{b}(j) \varepsilon-R(j)>\Delta E_{b}(k) \varepsilon-R(k) \Rightarrow \\
& \Delta E_{b}(j) \varepsilon-R(i)>\Delta E_{b}(k) \varepsilon-R(k)+\underbrace{(R(j)-R(i))}_{<0}
\end{aligned}
$$

We can see that depending on the difference between the first and second utility gains for the base station and first and second highest price requests, the base station may end up with a lower than second highest utility gain. 
Further, consider the maximization of the utility for user $j$ :

$$
\begin{aligned}
& \Delta E_{b}(j) \varepsilon-R(j)=\Delta E_{b}(j) \varepsilon-R(i)-R(j)+R(i)>0 \Rightarrow \\
& \Delta U_{b}=\Delta E_{b}(j) \varepsilon-R(i)>\underbrace{R(j)-R(i)}_{<0} \Rightarrow
\end{aligned}
$$

The final base station gain is not guaranteed to be positive.

Based on the above theorem, we see that the Vickrey auction implementation is not an appealing strategy for the base station operators. We propose an alternate strategy for rewarding users, a modified Vickrey auction, for which the payment for the winner node is the requested reward plus the difference between the first and second highest utility gains.

Theorem 2: For the proposed modified Vickrey auction with two-dimensional bids, paying the winner the above pricing scheme yields an effective gain for the BS that is equal to the second highest utility gain for the bidding.

Proof: Consider user $j$ to be the winner of the auction, and user $k$ to be the node that yields the second highest utility gain for the base station. Denote by $x$ the difference between the highest and second highest utility gains. Then

$$
\begin{aligned}
\Delta U_{b} & =\Delta E_{b}(j) \varepsilon-R(j)-x= \\
& =\Delta E_{b}(j) \varepsilon-R(j)+\Delta E_{b}(j) \varepsilon-R(j)+\Delta E_{b}(k) \varepsilon-R(k)= \\
& =\Delta E_{b}(k) \varepsilon-R(k)=\Delta E_{b}(k) \varepsilon-R(k)
\end{aligned}
$$

Theorem 3: For the proposed modified Vickrey auction with two-dimensional bids, the above described pricing strategy ensures that all users have a dominant strategy of bidding their true valuation of resources.

Proof: The first component of the two-dimensional bid for the players comprises the users' estimates of the energy gains they can provide for the base station. For this component, the users cannot cheat, as this is the fixed resource they are selling to the $\mathrm{BS}$. For the second component, pricing, users may potentially have incentives to cheat to obtain greater utilities.

Assume that node $j$ tries to cheat and departs from its true valuation bidding. Denote the node's true valuation by $\theta(j)$.

- Suppose that the node decides to bid $R(j)=\theta(j)+y$.

We consider the two possible cases:

a) Node $j$ loses the auction, which results in $U_{R}(j)=0$. If the node would have won the auction by bidding $R(j)=$ $\theta(j)$ then its utility would bave been $U_{R}(j)=\theta(j)+x, x>0$ So overbidding decreases its utility. If bidding the true valuation would not have won the auction, then $U_{R}(j)$ remains the same which implies that there is no incentive to overbid. b) Node $j$ wins the auction

In this case the node gets paid $R(j)=\theta(j)+x$, where $x^{\prime}$ is the difference between its utility gain and the next highest one (for node $\mathrm{k}$ ). If node $j$ would have won with a reduced price $R(j)=\theta(j)$ as well, the reward would have been $R(j)=$ $\theta(j)+x$. Given that the strategies of other users stay the same, we have

$$
x^{\prime}=\Delta E_{b}(j) \varepsilon-\theta(j)-y-\Delta E_{b}(k) \varepsilon-R(k)=x-y,
$$

which implies that node $j$ gets a reward of $R(j)=\theta(j)+y+x-y$ $=\theta(j)+x$ and in turn that node $j$ cannot increase its utility by raising its price above its true valuation.

- Suppose that node $j$ decides to underbid its true valuation: say, $R(j)=\theta(j)-y$.

We again consider two possible cases:

a) Node $j$ loses the auction so that $U_{R}(j)=0$, which implies that the lower bid does not change the outcome of the auction, and hence node $j$ does not have an incentive to underbid its true valuation of resources.

b) Node $j$ wins the auction

If the action of underbidding changed the outcome of the auction, then the payment: $R(j)=\theta(j)-y+x$, where $x^{\prime}$ is the difference between node $j$ 's utility gain and the next highest one.

If node $j$ would have bid its true valuation, then the difference in the utility gain for the BS between the winning node $k$ and node $j$ would have been $x$.

If node $j$ 's action changed the outcome of the auction in its favor (i.e., node $j$ wins) by underbidding, then node $j$ gets as a reward $R(j)=\theta(j)-y+x$, where $x^{\prime}$ can be determined as

$$
\begin{aligned}
& x^{\prime}=\Delta E_{b}(j) \varepsilon-R(j)+y-\Delta E_{b}(k) \mathcal{E}-R(k)=y-x, \\
& \text { with } x=\Delta E_{b}(k) \mathcal{E}-R(k)-\left(\Delta E_{b}(j) \mathcal{\varepsilon}-R(j)\right)>0 .
\end{aligned}
$$

Then the actual reward for node $j$ can be determined to be $R(j)=\theta(\mathrm{j})-y+y-x<\theta(j) \Rightarrow U_{R}(j)<0$.

Similarly, if underbidding wins the auction, but bidding true valuation would also have won, the node utility is $U_{R}(j)$ $=x^{\prime}-y$, with

$$
x^{\prime}=\Delta E_{b}(j) \varepsilon-R(j)+y-\Delta E_{b}(k) \varepsilon-R(k)=y+x,
$$

which implies $U_{R}(j)=x$, which does not increase with underbidding and thus node $j$ does not have an incentive to underbid its true valuation of resources.

\section{B. Effective Green Energy Savings}

While the above proposed auctioning mechanisms ensure that both BS operators and terminals experience utility gains, we need to show under what conditions these individual utility gains result in real overall energy savings and are not merely a shift of energy consumption from the base station to the mobiles. 
Assume that the true valuation of an arbitrary mobile node $j$ for its energy expenditure can be captured by a price per unit energy parameter $\mu(j)$, such that $\theta(j)=E_{b}{ }^{\prime}(j) \mu(j)$.

In order to achieve overall energy savings, when a node $j$ wins the auction, we need to satisfy the following condition:

$$
\Delta E_{b}(j)-E_{b}^{\prime}(j)>0
$$

If node $j$ wins the auction then the base station has a positive gain in utility:

$$
\begin{aligned}
\Delta U_{b}= & \Delta E_{b}(j) \mathcal{E}-E^{\prime}{ }_{b}(j) \mu(j)-x>0 \Rightarrow \\
& \Delta E_{b}(j)-E^{\prime}{ }_{b}(j) \mu(j) / \varepsilon-\underbrace{x / \varepsilon}_{>0}>0 \Rightarrow \\
& \Delta E_{b}(j)-E^{\prime}{ }_{b}(j) \mu(j) / \varepsilon>0 .
\end{aligned}
$$

If $\mu(j) / \varepsilon>1$, or equivalently, $\mu(j)>\varepsilon$, the above inequality guarantees effective green energy savings.

Thus, for a given cost per unit energy resource, $c$, we require

$$
\mu(j)>\delta=\max \{c, \lambda-c\} .
$$

We can define $\delta$ to be the reserve price for the relaying terminals, such that no resource is sold under this reserve price. The reserve price $\delta$ ensures true net energy savings for the system.

\section{IMPLEMENTATION ISSUES AND CONCLUSIONS}

The proposed auctioning mechanism ensures that overall effective energy savings can be implemented for the cellular systems by combining signal processing, with energy efficient resource management, relaying, and pricing.

Relaying has been shown in the literature to result in performance gains and/or energy savings for cellular networks (see for example [7] and the references therein).

In this paper we assume that each link requires a certain BER (bit error rate) performance (which, in conjunction with error correcting codes, is directly linked to the probability of correct packet reception in the utility function definitions). The required BER can be mapped into an SINR target $\gamma$. For an illustrative example for 3G wireless systems based on CDMA technology, the achieved target SINR requirement for a general linear receiver filter is given as:

$$
\operatorname{SINR}_{i}=\frac{P_{t} h_{B i}\left(c_{i}^{T} s_{i}\right)^{2}}{\left(c_{i}^{T} c_{i}\right) \sigma^{2}+\sum_{k \neq i} P_{k} h_{k i}\left(c_{i}^{T} s_{k}\right)^{2}}=\gamma,
$$

where $c_{i}$ is the receiver vector filter, $s_{i}$ is the signature sequence for user $i, h_{i j}$ is the path gain between transmitter $i$ and receiver $j$, and $\sigma^{2}$ is the noise power.

Hence, for a fixed transmission rate, the energy per bit gains that a mobile terminal can induce at the BS, are given by the power transmission gains due to link quality improvements:

$$
\Delta \hat{P}_{t}=\hat{P}_{t}-P_{t}^{d}=\gamma \frac{\left(c_{i}^{T} c_{i}\right) \sigma^{2}+\sum_{k \neq i} P_{k} h_{k i}\left(c_{i}^{T} s_{k}\right)^{2}}{h_{B i}\left(c_{i}^{T} s_{i}\right)^{2}}-P_{t}^{d} .
$$

The energy improvements $\Delta \hat{E}_{b}$ can be estimated by a potential relaying terminal by assessing $\Delta P_{t}$, based on initial knowledge of transmitted power required by the BS for the direct connection, $P_{t}^{d}$ (broadcast by the BS with the call set-up request at the auction initiation phase), and by estimating the downlink path gain for the new transmission and the current interference level at terminal, together with the impact of possible improvements from signal processing (e.g., multiuser detection mitigating the interference).

The performance/complexity tradeoff is captured by the computational energy $q$, which can be approximated based on the computational complexity of the algorithm [11].

The auctioning scheme in this paper has proposed the framework to enforce energy gains into cellular networks. More work is needed to develop specific implementations and to analyze and simulate the performance for this pricing scheme in combination with various resource allocation and signal processing algorithms.

\section{REFERENCES}

[1] O. Naomi, "Beyond the Ivory Tower: The Scientific Consensus on Climate Change," 5702, s.1. : Science, December 2004, Vol. 306, p. 1686.

[2] J. Hansen, M. Sato, P. Kharecha, G. Russell, D. Lea, M. Siddal, "Climate change and trace gases," Philosophical Transactions of Royal Society, May 2007, Vol. 365, pp. 1925-1954.

[3] Peter Grant, "Green Radio - The Case for More Efficient Cellular Base Stations," University of Edinburgh, http://www.see.ed.ac.uk/ pmg/.

[4] Green Radio.Wireless@KTH. [Online] Royal Institute of Technology (KTH). http://www.wireless.kth.se/projects/GREEN/index.php.

[5] H. Karl, "An overview of energy-efficiency techniques for mobile communication systems," Telecommunication Networks Group, Technical University Berlin, Tech. Rep. TKN-03-XXX, September 2003.

[6] J. Huang, Z. Han, M. Chiang and H. V. Poor, "Auction-based Resource Allocation for Cooperative Communications," IEEE Journal on Selected Areas in Communications - Special Issue on Game Theory in Communication Systems, Vol. 26, No. 7, pp. 12261237, September 2008.

[7] C. Raman. G.J. Foschini, R.A. Valenzuela, R.D. Yates and N.B. Mandayam, "Power Savings from Half-Duplex Relaying in Downlink Cellular Systems", to appear Proc. IEEE Globecom 2009.

[8] C. Saraydar, N. B. Mandayam and D. J. Goodman, "Power Control via Pricing in Wireless Data Network," February 2002, IEEE Trans. on Communications, Vol. 50, no 2, pp. 291-303.

[9] J. Huang, R. Berry, and M. L. Honig, "Auction-based Spectrum Sharing," Mobile Networks and Applications, vol. 11, no. 3, pp. 405418, June YEAR??

[10] V. Krishna, Auction Theory, Academic Press, London, UK, 2002.

[11] S.M. Futaci, K. jaffres Runser, C. Comaniciu, "On Modeling EnergySecurity Tradeoffs for Distributed Monitoring in Wireless Ad Hoc Networks," Proc. IEEE Conference on Military Communications (MILCOM 2008), San Diego, CA, November 2008.

[12] O. Ileri, S-C. Mau, N. B. Mandayam, "Pricing for Enabling Forwarding in Self-Configuring Ad hoc Networks," IEEE Journal on Selected Areas in Communications - Special Issue on Wireless Ad Hoc Networks, vol. 23, No. 1, pp. 151-162, January 2005. 